Nervenarzt 2022 · 93:405-409

https://doi.org/10.1007/s00115-021-01190-1

Angenommen: 16. Juni 2021

Online publiziert: 29 . September 2021

๑) Der/die Autor(en) 2021

\section{Dysphasische Anfälle infolge einer chronischen Leptomeningitis}

\section{Eine differenzialdiagnostische Herausforderung}

\author{
K. Olaciregui Dague' · J. Pukropski' · C. Hummel ${ }^{2}$ A. Becker ${ }^{2} \cdot$ R. Surges ${ }^{1}$. \\ T. Baumgartner ${ }^{1}$ \\ ${ }^{1}$ Klinik und Poliklinik für Epileptologie, Universitätsklinikum Bonn, Bonn, Deutschland \\ ${ }^{2}$ Institut für Neuropathologie, Sektion für Translationale Epilepsieforschung, Universitätsklinikum Bonn, \\ Bonn, Deutschland
}

\section{Fallbeschreibung}

Die 80-jährige Patientin stellte sich zur diagnostischen Zuordnung rezidivierender Episoden mit Sprachstörung in unserer Klinik vor. Erstmalig sei es vor ca. 6 Monaten zu einer Episode mit Wortfindungs- und Sprachverständnisstörungen für wenige Minuten gekommen. Die erste Episode sei von Parästhesien im rechten Arm begleitet gewesen. Daraufhin erfolgte eine Aufnahme in einer auswärtigen Klinik unter dem Verdacht einer transitorischen ischämischen Attacke. In der Magnetresonanztomographie (MRT) des Neurokraniums fand sich kein Hinweis auf eine frische Ischämie. Es zeigte sich jedoch eine kontrastmittelaufnehmende Verdickung der Leptomeninx links temporookzipital. In der daraufhin durchgeführten Liquordiagnostik fanden sich zunächst unauffällige Parameter (Zellzahl, Glukose, Eiweiß und Laktat in der Norm). Eine intrathekale Immunglobulinsynthese war nicht nachweisbar. Die Erregerdiagnostik zeigte ebenfalls keine Auffälligkeiten im Liquor (Borrelien, neurotrope Viren: HSV1/2, Masern, Röteln, VZV). In der Röntgenaufnahme des Thorax fand sich neben dem bekannten Situs inversus eine ovaläre Verschattung parahilär links mit einem Durchmesser von ca. $15 \mathrm{~mm}$. Die Patientin wurde zunächst mit ASS $100 \mathrm{mg} / \mathrm{Tag}$ in der Annahme einer transitorischen ischämischen Attacke entlassen. Etwa 5 Monate später ereigneten sich zwei weitere Episoden mit
Sprachstörung, weswegen die Patientin erneut in derselben Klinik vorstellig wurde. Magnetresonanztomographisch fand sich eine zunehmende Ausdehnung der kontrastaufnehmenden Verdickung der Leptomeninx linkshemisphärisch. Liquorchemisch war eine geringe Pleozytose (6 Leukozyten/ $\mu$; Normwert: $<5 / \mu \mathrm{l}$ ) und eine geringe Eiweißerhöhung $(501 \mathrm{mg} / \mathrm{l}$; Normwert 150-450 mg/l) nachweisbar. Im Reiber-Schema bestand eine intrathekale $\lg \mathrm{A}$ - und IgM-Antikörpersynthese. Tumorzellen fanden sich im Liquor nicht. Unter der Annahme rezidivierender fokaler Anfälle wurde eine antikonvulsive Therapie mit Levetiracetam etabliert.

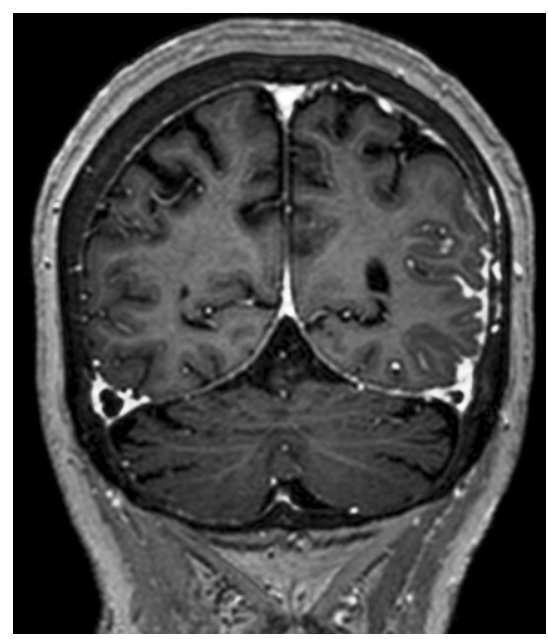

Abb. 1 \ Kraniale Magnetresonanztomographie (T1 mit Kontrastmittel) bei Aufnahme in unserer Klinik. Deutliche leptomeningeale Kontrastmittelaufnahme links temporoparietookzipital 


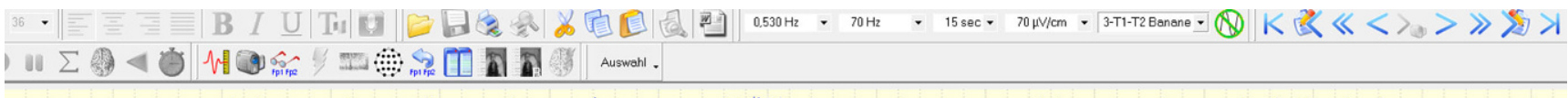

(n)

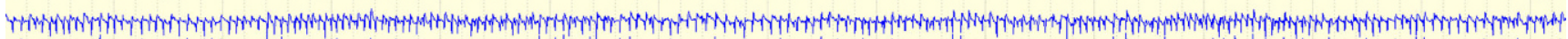

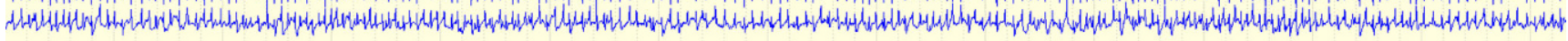
(n)

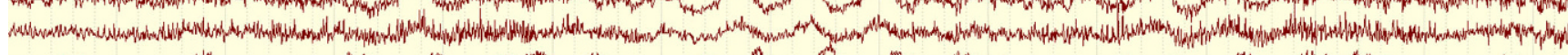
(n) (n)

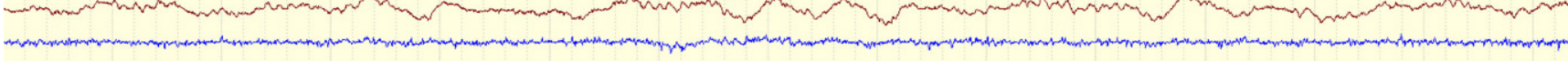
-

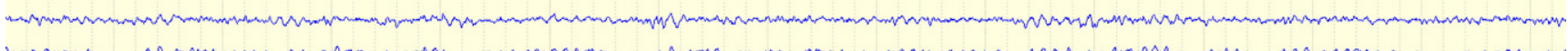

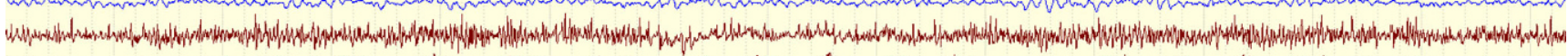

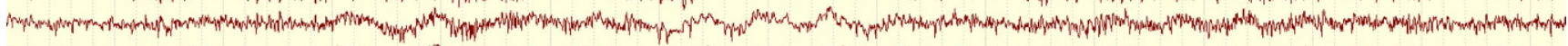

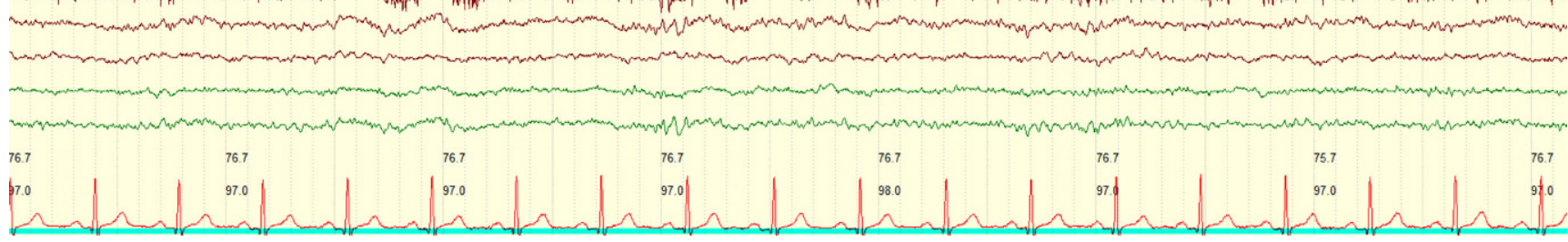

Abb. 2 \ Elektroenzephalographie bei Aufnahme in unserer Klinik. Delta-Verlangsamungsherd links temporookzipital, teils im Sinne einer rhythmischen Delta-Aktivität

Zur Vorgeschichte ist zu berichten, dass die Patientin an einer seropositiven (Rheumafaktor und Anti-CCP-Antikörper) rheumatoiden Arthritis leidet (Erstdiagnose 2000). Es wurde zunächst eine Basistherapie mit Methotrexat etabliert, die aufgrund von Blutbildveränderungen auf Leflunomid umgestellt wurde. Bei unzureichender Krankheitskontrolle wurde die Basistherapie im Jahr 2015 auf Certolizumab umgestellt, welche in Kombination mit Prednisolon 2,5 mg/die bis zur Aufnahme in unserer Klink fortgeführt wurde.

Bei der ersten Vorstellung in unserer Klinik zeigte die Patientin bis auf leichte Wortfindungsstörungen kein fokalneurologisches Defizit. Unter einer Therapie mit Levetiracetam hatten sich in der Zwischenzeit zwei weitere Episoden mit Sprachstörung ereignet. In der MRT des Neurokraniums stellte sich ein nahezu konstantes Bild der leptomeningeal betonten Kontrastmittelanreicherung dar (- Abb. 1). Bildgebende Hinweise auf eine zerebrale Amyloidangiopathie zeigten sich nicht. Eine ergänzende MRT des Myelons erbrachte keine Auffälligkeiten. In der Video-EEG-Ableitung zeigte sich ein kontinuierlicher Theta-Delta-Verlangsamungs- herd links temporookzipital (• Abb. 2). Liquorchemisch fanden sich eine intrathekale Drei-Klassen-Synthese (IgG, IgA, IgM) und eine lymphozytäre Pleozytose von 24 Zellen/ $\mu$ l (Normwert: $<5 / \mu \mathrm{l}$ ). Eine erneute umfangreiche virologische und mikrobiologische Erregerdiagnostik in Serum und Liquor mit Fokus auf atypische Erreger blieb ergebnislos (Mykobakterien, Borrelien, Spirochäten, Cryptococcus neoformans, Brucella spp., Nocardia spp.). Neuropathologisch gelang weiterhin kein Tumorzellnachweis im Liquor. Rheumafaktor $(129,7 \mathrm{IU} / \mathrm{ml}$, Normwert $<14 \mathrm{IU} / \mathrm{ml})$ und Anti-CCP-Antikörper ( $>2500 \mathrm{U} / \mathrm{ml}$, Normwert $<17 \mathrm{U} / \mathrm{ml}$ ) waren deutlich erhöht. C-reaktives Protein, Angiotensin Converting Enzyme, Interleukin-2-Rezeptor, antinukleäre Antikörper sowie zytoplasmatische und perinukleäre antineutrophile zytoplasmatische Antikörper waren im Normbereich. Eine weiterführende Diagnostik mittels Computertomographie (CT) des Thorax erbrachte mehrere partiell verkalkte Rundherde in beiden Lungenfeldern mit einer maximalen Größe von $11 \times 15 \mathrm{~mm}$. Eine Biopsie eines Lungenrundherdes mittels Bronchoskopie gelang nicht. Die mikrobiologischen Befunde der bronchoalveolären Lavage erbrach- ten keine Auffälligkeiten. Aufgrund einer Zunahme der leptomeningealen Kontrastmittelanreicherung 2 Wochen später erfolgte die Durchführung einer Hirnbiopsie links parietal. Im neuropathologischen Bericht zeigte sich das Bild einer zerebralen Amyloidangiopathie mit Nachweis leptomengingeal akzentuiert imponierender, floride-entzündlicher Infiltrate (• Abb. 3). Ein Erreger- oder Tumornachweis erfolgte auch in der Gehirnbiopsie nicht.

\section{Therapie und Verlauf}

Nach Vorstellung der Patientin in unserer Klinik wurde zunächst die antikonvulsive Therapie um Lacosamid erweitert. Zudem wurde die Therapie mit Certolizumab pausiert. In den darauffolgenden 3 Wochen wurden keine Anfälle mehr beobachtet. Jedoch zeigte sich die Sprachstörung fortschreitend, und die Patientin entwickelte ein leichtes Delir. Aufgrund des fortschreitenden klinischen und radiologischen Befundes erfolgte auch bei fehlendem Erregernachweis eine kalkulierte antibiotische Therapie mit Vancomycin und Meropenem. Hierunter kam es jedoch zu keiner Besserung des klinischen Befundes, so dass die Therapie bei ebenfalls fehlendem Erre- 




Abb. $3<$ a Eine immunhistochemische Untersuchung mit Antikörpern gegen Amyloid beta ergibt akzentuierte Markierungsmuster von Gefäßwandstrukturen (schwarze Pfeile). Innerhalb angrenzenden Gehirnparenchyms sind keine begleitenden Plaque-Strukturen zu erkennen. $b$ In einer Hämatoxylin und Eosin (HE) Färbung finden sich perivaskulär ausgeprägte Entzündungsinfiltrate (schwarzer Pfeil), die von Partien angrenzenden Gehirnparenchyms recht gut abgegrenzt erscheinen. Das Immunzellfiltrat besteht überwiegend aus T-Lymphozyten mit CD3- (c; sternförmige Markierung) sowie teils auch CD8- (d; sternförmige Markierung/Insert) Positivität, sowie CD68-positiven Makrophagen und entsprechend positiver begleitender Mikroglia (e). Man findet zudem schüttere CD20-exprimierende B-Lymphozyten (schwarzer Pfeil/Insert; f) sowie CD138-positive Plasmazellen (schwarzer Pfeil) Insert; $\mathbf{g}$ ) perivaskulär arrangiert (Balken $150 \mu \mathrm{m}$ in a, sowie in den Inserts zud, f, gund $500 \mu \mathrm{m}$ in b-g)

gernachweis in der Hirngewebsdiagnostik beendet wurde.

Etwa 6 Wochen nach der ersten Vorstellung in unserer Klinik erfolgte eine Kortisonstoßtherapie über 3 Tage (Methylprednisolon $500 \mathrm{mg} / \mathrm{Tag}$ ) und eine anschließende orale Kortisontherapie (Prednisolon
$1 \mathrm{mg} / \mathrm{kg} / \mathrm{KG})$. Bereits eine Woche nach Beginn der Kortisontherapie kam es zu einer deutlichen Befundbesserung in einer zerebralen Kernspintomographie (• Abb. 4). Zwei Monate später war keine leptomeningeale Kontrastmittelaufnahme mehr nachweisbar, der Liquor zeigte normwertige
Befunde, und Episoden mit Sprachstörung traten nicht mehr auf.

\section{Diskussion}

Der vorgestellte Fall demonstriert das breite differenzialdiagnostische Spek- 


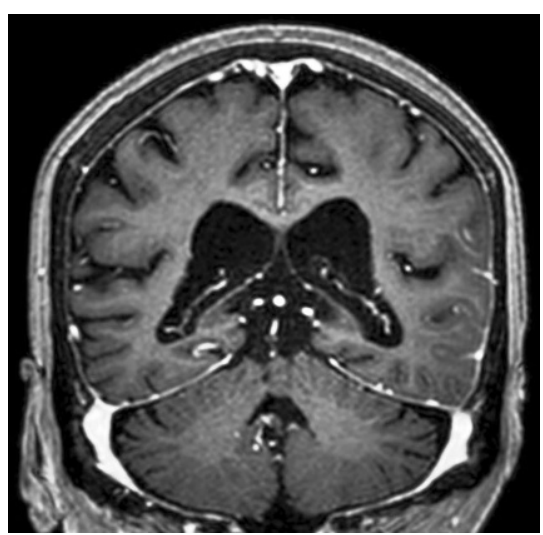

Abb. $4 \Delta$ Kraniale Magnetresonanztomographie (T1 mit Kontrastmittel) 7 Tage nach Beginn der Kortisontherapie

trum einer chronischen Leptomeningitis (• Tab. 1).

Im Fall unserer Patientin wurde aufgrund der langjährigen immunsuppressiven Therapie und des zunächst subakuten Krankheitsprogresses insbesondere eine atypische, erregerbedingte Ursache in Betracht gezogen. Unter Berücksichtigung der unklaren Lungenrundherde wurden neben dem Vorliegen eines Bronchialkarzinoms oder einer tuberkulösen Meningitis auch seltene opportunistische Erreger wie u. a. Nokardien diskutiert. Insbesondere die Diagnosestellung einer tuberkulösen Meningitis kann Behandler vor große Schwierigkeiten stellen, da sowohl die PCR aus dem Liquor (Sensitivität: 50-60\%) als auch der mikroskopische Nachweis von säurefesten Stäbchen (Sensitivität: 10-15\%) eine geringe bis mäßige Sensitivität zeigen. Der kulturelle Nachweis dauert zudem mehrere Wochen [1]. Weitere Erreger (insbesondere Mykosen, Brucellose, neurotrope Viren) mussten ebenfalls differenzialdiagnostisch berücksichtigt werden. Hinweise auf eine Neurosarkoidose fanden sich laborchemisch nicht.

Zudem wurde eine aseptische Meningitis infolge der TNF-Blocker-Therapie mit Certolizumab diskutiert. Mehrere Einzelfallberichte einer aseptischen Meningitis unter verschiedenen TNF-Blockern sind in der Literatur beschrieben [3, 5]. Neben den o. g. Differenzialdiagnosen wurde eine rheumatoide Meningitis favorisiert. Die zum Zeitpunkt des Erkrankungsbeginns fehlende Krankheitsaktivität der rheumathoiden Arthritis andere Organsysteme betreffend und das Fehlen systemischer Ent-

Tab. 1 Differenzialdiagnosen der chronischen Meningitis

\begin{tabular}{|l|l|}
\hline Chronische Meningitis \\
\hline Septisch & $\begin{array}{l}\text { Bakterien: Myokobakterien (insbesondere Mycobacterium tuberculosis), Nokardi- } \\
\text { en, Brucellose, Treponema pallidum, Borrelia burgdorferi }\end{array}$ \\
\cline { 2 - 3 } & Pilze: insbesondere Cryptococcus neoformans, Aspergillus, Candida \\
\cline { 2 - 3 } & Parasiten: Toxoplasma gondii \\
\cline { 2 - 3 } & Viren: Enteroviren, HSV 1 und 2, HHV 6, VZV \\
\hline & $\begin{array}{l}\text { Meningeosis carcinomatosa } \\
\text { Lupus erythematodes, Morbus Behçet }\end{array}$ \\
\cline { 2 - 3 } & $\begin{array}{l}\text { Weitere immunologische Erkrankungen: Neurosarkoidose, primäre ZNS-Vaskulitis, } \\
\text { ZNS-Beteiligung bei systemischer Vaskulitis }\end{array}$ \\
\cline { 2 - 3 } & $\begin{array}{l}\text { Medikamentös induziert: IVIGs, Monoklonale Antikörper, nichtsteroidale Antirheu- } \\
\text { matika, Methotrexat }\end{array}$ \\
\cline { 2 - 3 } & Andere Ursachen: zerebrale Amyloidangiopathie assoziiert mit Inflammation \\
\hline
\end{tabular}

zündungszeichen, ließ jedoch zunächst an der Diagnose zweifeln.

Letztendlich wurde sich aufgrund der fortschreitenden Klinik insbesondere zum Ausschluss 1) einer fokalen Meningeosis carcinomatosa (auch bei mehrfach fehlendem Tumornachweis im Liquor), 2) einer zerebralen Vaskulitis und 3) einer tuberkuIösen Meningitis für eine Hirnbiopsie entschieden. Der neuropathologische Befund erbrachte ein Bild, welches mit einer zerebralen Amyloidangiopathie assoziiert mit Inflammation („,cerebral amyloid angiopathy-related inflammation", CAA-RI) vereinbar war. Auch wenn die zerebrale MRT (kein Nachweis einer superfiziellen Siderose, nur einzelne Microbleeds) nicht das typische Bild einer zerebralen Amyloidangiopathie zeigte, war eine CAA-RI mit initialer leptomeningealer Affektion nicht ausgeschlossen (s. auch [2]).

Nach bestmöglichem Ausschluss einer infektiös erregerbedingten Genese wurde sich für eine Kortisonstoßtherapie in der Annahme einer rheumatoiden Meningitis entschieden. Das schnelle und sehr gute Ansprechen unterstützte klar diese Diagnose. Den histologischen Nachweis der zerebralen Amyloidangiopathie interpretieren wir rückblickend als koinzidenten Befund einer in dieser Altersgruppe nicht seltenen Pathologie [4] ohne sicheren Zusammenhang mit dem aktuellen Krankheitsgeschehen.
Korrespondenzadresse

\section{Dr. K. Olaciregui Dague}

Klinik und Poliklinik für Epileptologie,

Universitätsklinikum Bonn

Venusberg-Campus 1, 53127 Bonn,

Deutschland

karmele.olaciregui_dague@ukbonn.de

Funding. Open Access funding enabled and organized by Projekt DEAL.

Interessenkonflikt. K. Olaciregui Dague, J. Pukropski, C. Hummel, A. Becker, R. Surges und T. Baumgartner geben an, dass kein Interessenkonflikt besteht.

Open Access. Dieser Artikel wird unter der Creative Commons Namensnennung 4.0 International Lizenz veröffentlicht, welche die Nutzung, Vervielfältigung, Bearbeitung, Verbreitung und Wiedergabe in jeglichem Medium und Format erlaubt, sofern Sie den/die ursprünglichen Autor(en) und die Quelle ordnungsgemäß nennen, einen Link zur Creative Commons Lizenz beifügen und angeben, ob Änderungen vorgenommen wurden.

Die in diesem Artikel enthaltenen Bilder und sonstiges Drittmaterial unterliegen ebenfalls der genannten Creative Commons Lizenz, sofern sich aus der Abbildungslegende nichts anderes ergibt. Sofern das betreffende Material nicht unter der genannten Creative Commons Lizenz steht und die betreffende Handlung nicht nach gesetzlichen Vorschriften erlaubt ist, ist für die oben aufgeführten Weiterverwendungen des $\mathrm{Ma}$ terials die Einwilligung des jeweiligen Rechteinhabers einzuholen.

Weitere Details zur Lizenz entnehmen Sie bitte der Lizenzinformation auf http://creativecommons.org/ licenses/by/4.0/deed.de.

\section{Literatur}

1. Bahr NC, Meintjes G, Boulware DR (2019) Inadequate diagnostics: the case to move beyond the bacilli for detection of meningitis due to Mycobacterium tuberculosis. J Med Microbiol 


\section{Fachnachrichten}

68(5):755-760. https://doi.org/10.1099/jmm.0. 000975

2. Coulette S, Renard D, Lehmann S, Raposo N, Arquizan C, Charif M, Thouvenot E, Wacongne A, Viguier A, Bonneville F, Allou T, Boukriche $Y$, Chiper L, Blanchet Fourcade G, Gabelle A, Ducros A, Duflos C, Labauge P, Menjot de Champfleur N, Ayrignac X (2019) A clinico-radiological study of cerebral amyloid angiopathy-related inflammation. Cerebrovasc Dis 48(1/2):38-44. https://doi. org/10.1159/000502832

3. Hamilton AJ, Gutowski NJ (2011) Aseptic meningitis in a patient receiving treatment with anti-tumour necrosis factor alpha. J Neurol 258(6):1174-1175. https://doi.org/10.1007/ s00415-010-5887-7

4. Keage HA, Carare RO, Friedland RP et al (2009) Population studies of sporadic cerebral amyloid angiopathy and dementia: a systematic review. BMC Neurol 9(2009):13. https://doi.org/10.1186/ 1471-2377-9-3

5. Manthey C, Lohse AW, Pace A (2011) Case report of aseptic meningitis in a patient with Crohn's disease under infliximab therapy. Inflamm Bowel Dis 17(2):E10. https://doi.org/10.1002/ibd.21324

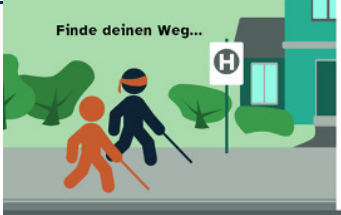

Ein Spiet der

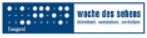

Aksion

\section{Blind zum Bus}

\section{Inklusives Online-Spiel "Blind zum Bus" simuliert Blindheit}

Wie findet man eigentlich seinen Weg zum Bus, wenn man nicht sehen kann? Hier können Sie es spielerisch ausprobieren.

Das inklusive Online-Spiel „Blind zum Bus" von der "Woche des Sehens" hilft Blindheit zu verstehen. Auf einem imaginären Gang bewegen sich die Spielenden von der Haustür bis zur Bushaltestelle. Das Besondere: Der Weg bleibt für alle unsichtbar, allein akustische Signale und Hinweise geben Anhaltspunkte, wo es lang geht.

\section{Spielablauf}

Aufgabe der Spielenden ist es, den Protagonisten zu steuern. Dieser folgt der Idee einer blinden Freundin und bewegt sich mit verbundenen Augen zum Bus. Bei der Orientierung helfen die Geräusche des Blindenlangstocks auf dem Pflaster und Straßengeräusche. Zudem gibt es Tipps von der blinden Freundin.

Der Weg zum Bus führt über fünf zunehmend komplexe Level. Für sehende Menschen eher ungewohnt, bleibt der Bildschirm bei „Blind zum Bus" weitgehend schwarz. Der Fokus liegt auf dem, was zu hören ist.

Nach jedem Level bekommen die Spielenden die Zahl der Versuche und die benötigte Zeit angezeigt. Zudem erhalten sie eine grafische Darstellung des zurückgelegten Weges. Da sich der Ausgangspunkt mit jedem Spielstart verändert, kann das Spiel beliebig oft wiederholt werden.

In einem zweiten Spiel "Zug in Sicht" lassen sich drei verschiedene Sehbehinderungen simulieren.

Beide Spiele sind barrierefrei online oder per App zu spielen und hier zu finden: https://www.woche-des-sehens.de/spiel 